\title{
CAPÍTULO 10: CRIAÇÃO DO PROJETO DE EXTENSÃO NÚCLEO DE IMPLANTE COCLEAR DA UNIVERSIDADE CATÓLICA DE PERNAMBUCO
}

\author{
CAPÍTULO 10: CREACIÓN DEL PROYECTO DE EXTENSIÓN NÚCLEO DE \\ IMPLANTES COCLEARES DEL UNIVERSIDADE CATÓLICA DE PERNAMBUCO
}

\section{CHAPTER 10: CREATION OF THE COCHLEAR IMPLANT CORE EXTENSION PROJECT OF UNIVERSIDADE CATÓLICA DE PERNAMBUCO}

\begin{abstract}
Pablo Vinicius do Nascimento Pinto ${ }^{1}$; Samuel Nascimento Neto ${ }^{2}$; Silvano Martins da Silva Filho ${ }^{3}$ Flávio Arthur Morais Cabral ${ }^{4}$; Cristiane Raquel Souto Zilbermintz ${ }^{5}$
\end{abstract}

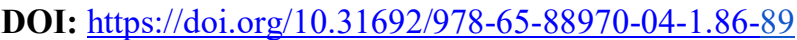

\section{INTRODUÇÃO}

A capacidade de um ouvinte humano identificar um som e a diversidade de eventos acústicos percebida, com intensidades e frequências distintas, é algo extraordinário (MENEZES E HYPPOLITO, 2015). A audição é o veículo no qual o indivíduo é imerso no mundo sonoro, no qual é possível experienciá-lo e constituir-se de maneira mais completa, Lima et.al (2004) nos diz que a linguagem pode ocorrer por intermédio de dois canais, sendo eles o canal auditivo, englobando a fala e sua compreensão e o canal visual, que é necessário para a leitura e escrita, para os gestos e consequentemente para a Língua de Sinais, utilizada pelos indivíduos surdos, afinal experienciamos o mundo através dos sentidos, possibilitando através das estruturas da linguagem, o desenvolver de um código próprio da espécie humana: a língua. Entretanto, há um grupo de pessoas, cuja deficiência e o tolher das capacidades auditivas organogênesicamente, lhes impede de experienciar o mundo através desse sentido, a audição.

De acordo com Dessen e Brito (1997), a deficiência auditiva é um tipo de privação sensorial, cujo sintoma comum é uma reação anormal diante do estímulo sonoro, por sua vez, a surdez é caracterizada por uma perda, maior ou menor, da percepção normal dos sons, havendo vários tipos de deficiência auditiva, em geral classificadas de acordo com o grau de perda da audição.

O Ministério da Saúde no ano de 2004 instituiu a Política Nacional de Atenção à Auditiva que preconiza a promoção à saúde auditiva, prevenção e a identificação precoce de

\footnotetext{
${ }^{1}$ Fonoaudiologia, Universidade Católica de Pernambuco, pabloviniciusdonp@gmail.com

${ }^{2}$ Fonoaudiologia, Universidade Católica de Pernambuco, samuelnasneto@gmail.com

${ }^{3}$ Psicologia, Centro Universitário Maurício de Nassau, silvanomartins614@gmail.co

${ }^{4}$ Psicologia, Universidade Católica de Pernambuco, flavioarthurmorais@gmail.com

${ }^{5}$ Mestra, Universidade Católica de Pernambuco, cristiane.zilbermintz@unicap.br
} 
problemas auditivos em ações específicas voltadas à saúde de gestantes, crianças, adolescentes, adultos e idosos (DAHER E PISANESCHI, 2010).

Dentre os procedimentos voltados à saúde auditiva, o Sistema Único de Saúde (SUS) oferece a cirurgia do implante coclear (IC), que segundo Bevilacqua (2004), se caracteriza por ser uma prótese computadorizada que é inserida cirurgicamente na cóclea, com o objetivo de substituir parcialmente as funções do órgão espiral, fornecendo impulsos elétricos para a estimulação direta das fibras neurais. Outrossim, os pacientes operados pelo SUS, em sua maioria, não têm atendimento contínuo e multidisciplinar, pós cirurgia, para sua reabilitação. O sujeito surdo agora implantando pode ouvir, mas irá precisar de ajuda clinica para o desenvolvimento e adaptação de suas recém-capacidades auditivas.

\section{RELATO DE EXPERIÊNCIA}

O projeto de extensão Núcleo de Implante Coclear da Universidade Católica de Pernambuco (NIC-UNICAP) é um projeto que abrangem profissionais e graduandos de Fonoaudiologia, Psicologia, Enfermagem, Medicina e Pedagogia, com o intuito de dar assistência aos surdos implantados, em sua maioria crianças, através do acompanhamento no pós-operatório.

O projeto segue o paradigma multidisciplinar e transdisciplinar. O paradigma multidisciplinar, para Bicalho e Oliveira (2011), corresponde à busca da integração de conhecimentos por meio do estudo de um objeto de uma mesma e única disciplina ou por várias delas ao mesmo tempo. Já o paradigma transdisciplinar, segundo Tanaka e Melo (2000) é compreendido como um paradigma pelo qual a avaliação pode ser empregada de forma a buscar, em conjunto, soluções para os problemas enfrentados. Exigindo um esforço coletivo de capacidades e aprendizagem para a ação, abertura do caminho para um exercício democrático na tomada de novas decisões, uma vez que, o trabalho é feito em equipe.

A idealização do projeto surgiu através das vivências da fonoaudióloga e audiolog Cristiane Zilbermintz, coordenadora do projeto, visando à atenção e acolhimento aos pacientes usuários de implante coclear que em sua maioria não têm acesso a serviços especializados na área de reabilitação auditiva. O projeto tem como principais objetivos: dar assistência a surdos implantados; promover autonomia da criança usuária de implante coclear audibilidade dos sons em geral; trabalhar sociabilização e desenvolvimento da linguagem e aprendizagem; possibilitar novas formas de manifestação subjetiva e; promover saúde mental aos pacientes e responsáveis. 
Busca-se oferecer um atendimento semanal, integral e transdisciplinar. Os pacientes passam por um circuito de atendimentos de fonoterapia, ajuda pedagógica, escuta e auxílio psicológico.

Há terapia musical durante as sessões uma vez que o ritmo e a melodia são as ferramentas mais ricas de estimulação de linguagem. Todos os atendimentos são discutidos em equipe no intuito de haver colaboração e melhor prognóstico do paciente, através dos conhecimentos, habilidades e competências da equipe multidisciplinar. Com a equipe multidisciplinar e transdisciplinar podemos desenvolver comunicação oral, linguagem receptiva e expressiva, a sociabilização, integração desses sujeitos do ponto de vista social, emocional e psíquico. Trabalhar o desenvolvimento das inteligências, emocional, linguística, musical, espacial, corporal e propriocepção global, além da metalinguagem, raciocínio lógico e o acolhimento familiar.

Figura 01: Equipe Multiprofissional do Projeto de Extensão do Núcleo de Implante Coclear da UNICAP, Recife-PE

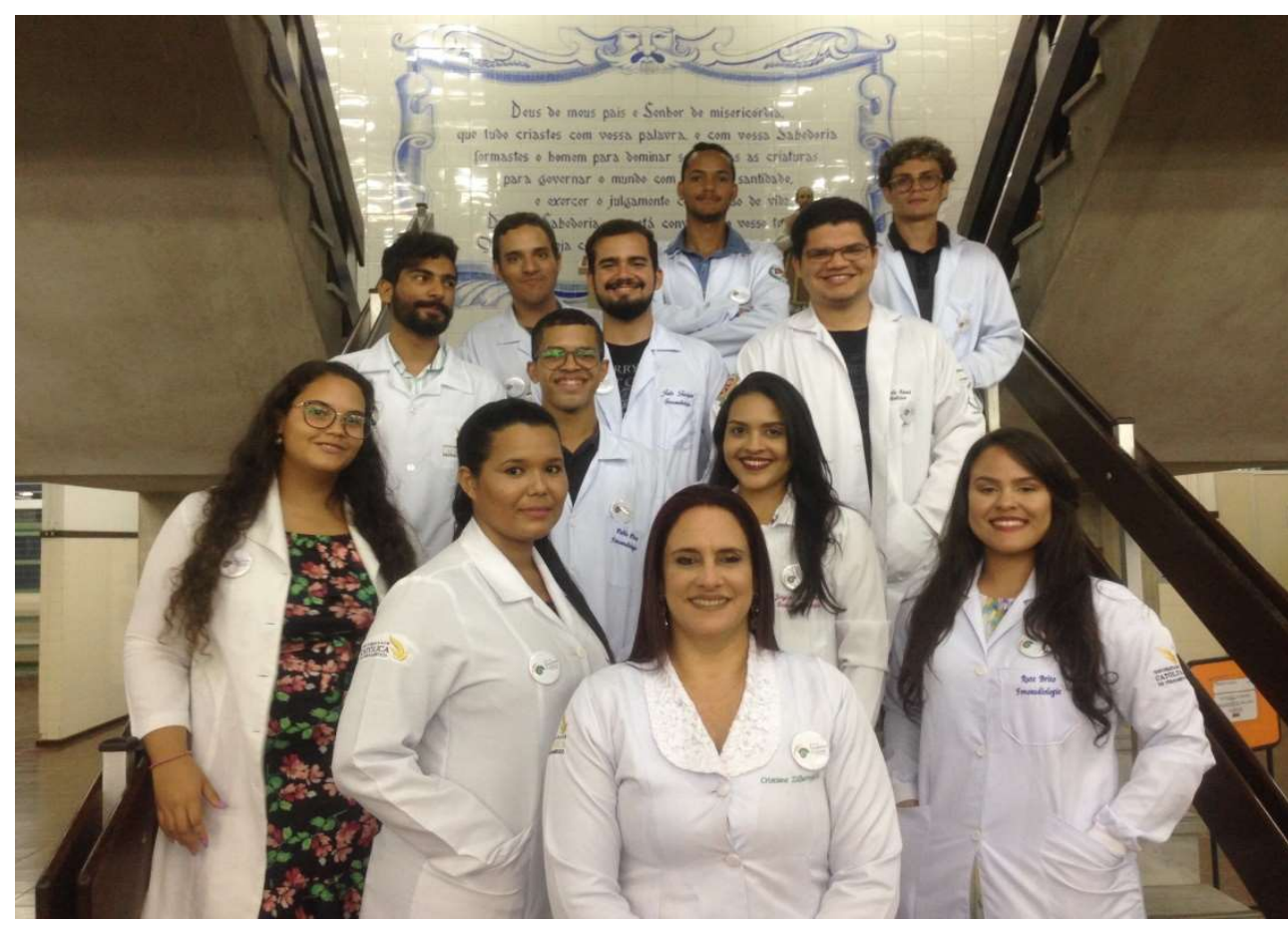

Fonte: FRANÇA (2019). 


\section{CONCLUSÕES}

$\mathrm{O}$ atendimento semanal, juntamente com a atuação transdisciplinar no atendimento às pessoas surdas usuárias de implante cloclear no Projeto de Extensão do Núcleo de Implante Coclear da Universidade Católica de Pernambuco permite que sejam trabalhadas diferentes perspectivas dos pacientes que corroboram mais efetivamente para o desenvolvimento desses sujeitos, gerando maior autonomia e sociabilidade através das inteligências adquiridas.

\section{REFERÊNCIAS}

BEVILACQUA, M.C. et.al. Impante Coclear. In: FERRIRA,L.P.; BEFI-LOPES, D.M.; LIMONGI, S.C.O. (Orgs). Tratado de fonoaudiologia. - $1^{\circ}$.ed. - São Paulo: Roca, 2004.

\section{BICALHO, L.M; OLIVEIRA, M. ASPECTOS CONCEITUAIS DA MULTIDISCIPLINARIDADE E DA INTERDISCIPLINARIDADE E A PESQUISA EM CIÊNCIA DA INFORMAÇÃO. Santa Catarina: Encontros Bibli, 2011.}

DAHER, C.V; PISANESCHI, E. A política nacional de atenção à saúde auditiva: a atenção especializada às pessoas com deficiência auditiva no SUS. In: BEVILACQUA, M.C. et.al. Saúde auditiva no Brasil: políticas, serviços e sistemas. São Paulo: Pulso Editorial, 2010.

DESSEN, M.A; BRITO, A.M.W. Reflexões sobre a deficiência auditiva e o atendimento institucional de crianças no Brasil. São Paulo: Paidéia (Ribeirão Preto), 1997.

FRANÇA, D. Clínica de Fonoaudiologia lança projeto para atender pessoas com implante coclear. Boletim UNICAP, 2019. Disponível em: $<$ http://www.unicap.br/assecom1/clinica-defonoaudiologia-lanca-projeto-para-atender-pessoas-com-implante-coclear/>. Acesso em: 19 de set. de 2020 .

LIMA, M.C.M.P. et.al. Observação do desenvolvimento de linguagem e funções auditiva e visual em lactentes. São Paulo: Revista de Saúde Pública, 2004.

MENEZES, P.L; HYPPOLITP, M.A. Biofísica da audição e bases para a Audiologia. In: BOÉCHAT, E.M. et.al (Orgs) Tratado de Audiologia - 2. ed. Rio de Janeiro: Guanabara Koogan, 2015.

TANAKA, O.Y; MELO, C. Uma proposta de abordagem transdisciplinar para avaliação em Saúde. São Paulo: Interface (Botucatu), 2000. 